\title{
Effect of contextual variables on mealtime problem behavior in the natural environment
}

\author{
Ellen J. McCartney \\ West Virginia University
}

Follow this and additional works at: https://researchrepository.wvu.edu/etd

\section{Recommended Citation}

McCartney, Ellen J., "Effect of contextual variables on mealtime problem behavior in the natural environment" (2004). Graduate Theses, Dissertations, and Problem Reports. 813.

https://researchrepository.wvu.edu/etd/813

This Thesis is protected by copyright and/or related rights. It has been brought to you by the The Research Repository @ WVU with permission from the rights-holder(s). You are free to use this Thesis in any way that is permitted by the copyright and related rights legislation that applies to your use. For other uses you must obtain permission from the rights-holder(s) directly, unless additional rights are indicated by a Creative Commons license in the record and/ or on the work itself. This Thesis has been accepted for inclusion in WVU Graduate Theses, Dissertations, and Problem Reports collection by an authorized administrator of The Research Repository @ WVU. For more information, please contact researchrepository@mail.wvu.edu. 
Effect of Contextual Variables on Mealtime Problem Behavior in the Natural Environment

\author{
Ellen J. McCartney
}

Thesis submitted to the Eberly College of Arts and Sciences at West Virginia University in partial fulfillment of the requirements for the degree of

\author{
Master of Arts in Psychology
}

Cynthia M. Anderson, Ph.D., Chair Michael Perone, Ph.D.

Lindsey L. Cohen, Ph.D.

Department of Psychology

Morgantown, West Virginia

2004

Keywords: Mealtime problem behavior, contextual or antecedent variables, assessment 


\begin{abstract}
Effect of Contextual Variables on Mealtime Problem Behavior in the Natural Environment
\end{abstract}

\author{
Ellen J. McCartney
}

A growing body of research suggests that contingency-based interventions are effective for decreasing mealtime problem behavior and increasing the amount and variety of food accepted. To date, two published studies have examined the effects of specific contextual variables (food type and texture or session pre-feeding) on mealtime behavior. Research with problem behavior occurring outside of mealtime suggests that problem behavior may often be under the control of contextual or contextual variables. The purpose of this study was to further evaluate the effect of contextual variables on mealtime behavior. Four children and their caregivers served as participants in both Phases 1 and 2. In Phase 1 preference and pre-feeding assessments were conducted. In Phase 2 participants were exposed to 3 or 4 contextual variables (e.g., preferred food present, tangible, idiosyncratic and other family members present) under conditions of food deprivation versus session pre-feeding. Across participants, the occurrence of mealtime problem behavior was found to decrease during the presentation of different contextual variable arrangements. 


\section{Table of Contents}

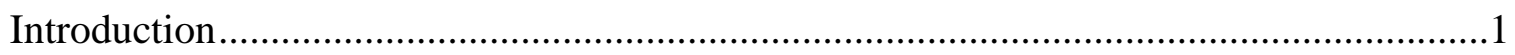

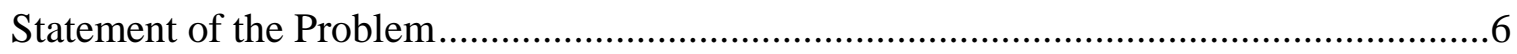

Phase 1: Preference and Pre-Feeding Assessments ....................................................

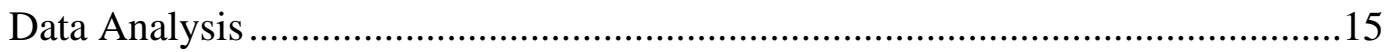

Results and Discussion ........................................................................ 15

Preference Assessment.................................................................15

Pre-Feeding Assessment ..............................................................17

Phase 2: Contextual Variable Evaluation ................................................................17

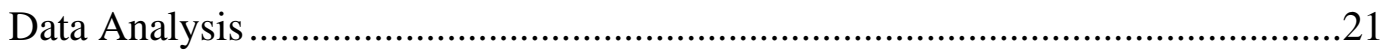

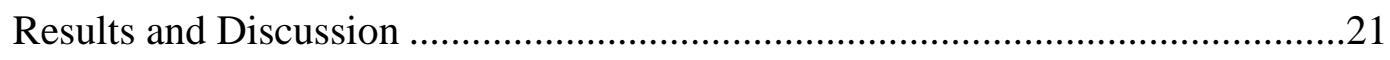

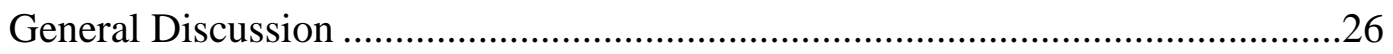




\section{List of Tables}

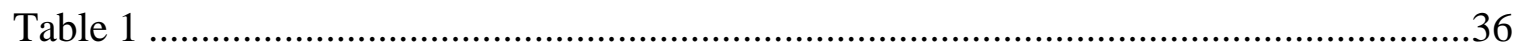

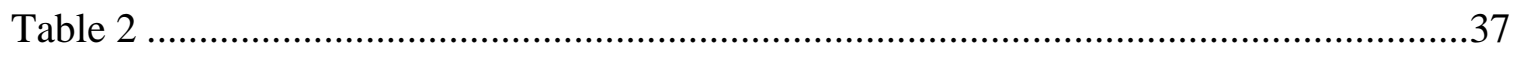

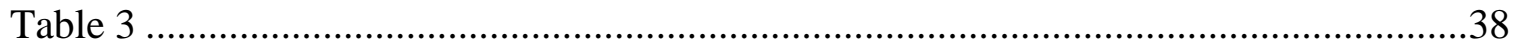

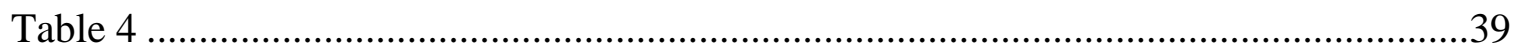




\section{List of Figures}

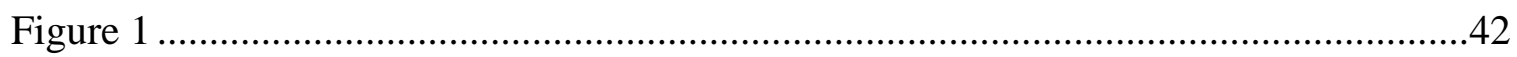

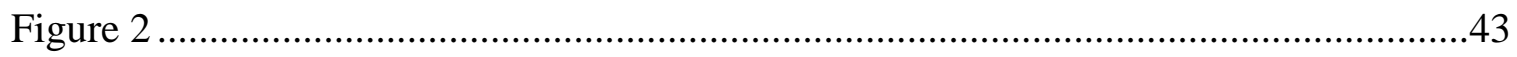

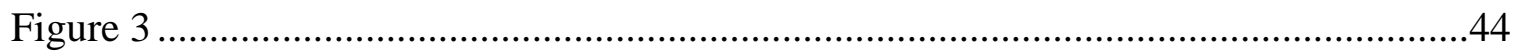

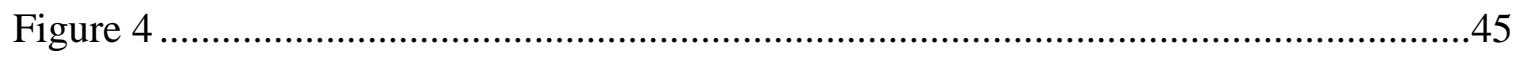

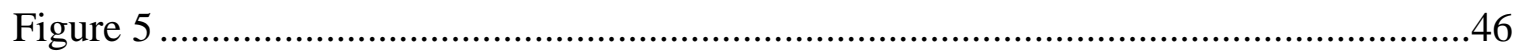

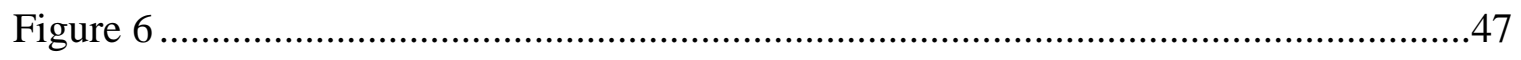

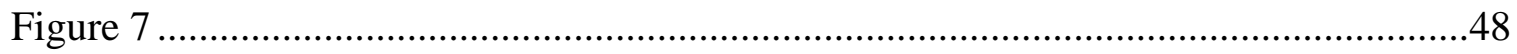

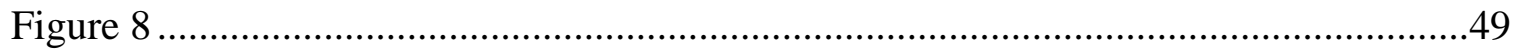

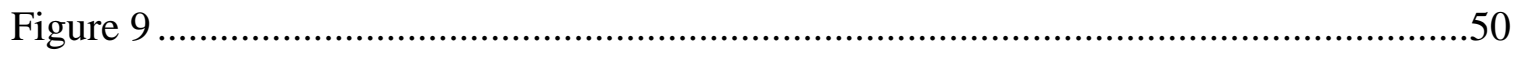


Effect of Contextual Variables on Mealtime Problem Behavior in the Natural Environment

Mealtime behavior problems (e.g., food refusal, food selectivity, disruptive behavior) are relatively common in the pediatric population; it is estimated that $25 \%$ to $35 \%$ of typicallydeveloping children, one-third of children with disabilities, and $80 \%$ of individuals with severe and profound mental retardation display such behavior (Palmer, Thompson, \& Linscheid, 1975; Riordan, Iwata, Wohl, \& Finney, 1980). Mealtime behavior problems can have a deleterious affect on children's physical, social, and educational development and also are associated with an inadequate intake of nutrients and weight loss (Kedesdy \& Budd, 1998).

Several variables have been linked to the development and exacerbation of mealtime difficulties including anatomical deficits and medical problems (e.g., gastro-intestinal reflux). Although organic impairment may result in mealtime difficulties, environmental factors often play a role in the development, maintenance or exacerbation of feeding problems. Research suggests that almost $20 \%$ of feeding problems are due solely to environmental factors (Babbitt, Hoch, Coe, \& Cataldo, 1994; Linscheid, Budd, \& Rasnake, 1995; Riordan, Iwata, Finney, Wohl, \& Stanley, 1984), which may develop concurrently with organic or medical problems, and often persist once such problems are ameliorated. Environmental factors responsible for the maintenance of mealtime difficulties may include reinforcement of problem behavior by allowing the child to escape from the meal situation or allowing a child to completely avoid eating certain foods and by delivering more preferred foods contingent on problem behavior (Babbitt et al., 1994; Riordan et al., 1984; Werle, Murphy, \& Budd, 1993). In addition, caregivers sometimes do not provide reinforcement contingent on appropriate behaviors such as acceptance of a bite of food. 
A growing body of research is focused on assessment and treatment of food selectivity and other mealtime behavior problems. Several different interventions have been developed to increase the amount and variety of foods consumed, including punishment (e.g., jaw prompting, Kerwin, Ahearn, Eicher, \& Burd, 1995), extinction (e.g., Hoch, Babbitt, Coe, Krell, \& Hackbert, 1994), and differential negative or positive reinforcement (e.g., Kitfield \& Masalsky, 2000; Patel, Piazza, Martinez, Volkert, \& Santana, 2002; Shore, Babbitt, Williams, Coe, \& Snyder, 1998). A multi-component intervention frequently reported in the literature is escape extinction (e.g., Cooper et al., 1995; Galensky, Miltenberger, Stricker, \& Garlinghouse, 2001; Hoch et al., 1994) which consists of differential reinforcement for appropriate behaviors and extinction for problem behaviors. Reinforcement for appropriate behavior such as acceptance of food typically includes both positive (e.g., attention, access to preferred tangibles) and negative (e.g., temporary escape from the eating situation, removal of spoon from child's lips) reinforcement. The extinction component involves withholding attention and escape or avoidance contingent on refusal and mealtime problem behavior.

Although behavioral interventions, and particularly escape extinction, have been demonstrated to be effective in increasing the amount or variety of food accepted (e.g., Ahearn, Kerwin, Eicher, Shantz, \& Swearingin 1996; Babbitt et al., 1994; Cooper et al., 1995; Didden, Seys, \& Schouwink, 1999; Hoch et al., 1994), only a limited number of studies have examined the efficacy and durability of behavioral interventions when implemented by typical caregivers (e.g., parents) in community settings. Of existing studies, many are difficult to interpret due to limitations such as a lack of treatment integrity data (e.g., Didden et al., 1999; O’Reilly \& Lancioni, 2001), subjective measures of the dependent variable (e.g., Luiselli, Evans, \& Boyce, 1985), or a limited number of subjects (e.g., Anderson \& McMillan, 2002). Werle and colleagues 
conducted two studies to evaluate parental implementation of behavioral interventions for feeding disorders (Werle, Murphy, \& Budd, 1993, 1998). Werle et al. demonstrated the efficacy of behavioral parent training for increasing appropriate mealtime behavior, but intervention components were not consistently implemented across participants (e.g., timeout was used with some but not all participants). In addition, parents were not specifically taught to continue presentation of non-preferred foods until acceptance occurred (i.e., escape extinction). Therefore, the intervention might not be as effective with children who exhibit severe food selectivity, food refusal, or high rates of problem behavior at mealtime.

Mueller et al. (2003) systematically evaluated methods of parent training for teaching parents to implement behavioral interventions for food selectivity. Working in an outpatient clinic, they compared review of written protocols, verbal instructions, therapist modeling, and rehearsal training. Mueller et al. found that verbal instruction, modeling, and rehearsal improved procedural integrity but written and verbal instruction alone had little effect. Because experimenters implemented the interventions initially (until clinically significant gains were made) it is not possible to determine whether parents would have been able to implement the intervention successfully from the outset. Further, no data were provided on the extent to which treatment gains were maintained when parents began feeding their children. One reason that research on community-based interventions for pediatric feeding problems is so scarce may be that escape extinction is a difficult treatment to implement with integrity. High rates of problem behavior (e.g., head turning, hitting the spoon, gagging, etc.) are common during initial treatment meals and the child typically appears extremely distressed as crying, screaming, and negative vocalizations are common. Anecdotally, parents who have attempted to implement escapeextinction often report that they "feel bad for their child” and “can’t take their child crying.” 
Further, data from these meals reveals that parents often do not completely ignore problem behavior, even after extensive training in the intervention. Parental willingness and ability to effectively implement escape extinction may be increased if the likelihood of problem behavior during initial treatment meals is reduced. Research on problem behavior in areas outside of mealtime suggests that contextual interventions may result in such an outcome.

\section{Contextual/Contextual Influences on Behavior}

Although mealtime problem behavior most often is maintained by positive or negative reinforcement, problem behavior may occur only under certain stimulus conditions, such as the presence of particular types of foods or certain individuals. One study has directly evaluated the effects of contextual stimuli on mealtime problem behavior. Munk and Repp (1994) examined the effects of different types (e.g., vegetables, meats) and textures (e.g., smooth, coarse) of foods on mealtime behavior problems emitted by five individuals with mental retardation exhibiting food selectivity or food refusal. Three individuals refused foods regardless of the type or texture of the food, while the remaining 2 individuals exhibited problem behavior only in the presence of certain types or textures.

In an additional investigation, Levin and Carr (2001) demonstrated that mild food deprivation was not enough to increase the likelihood that food selective children will accept bites of non-preferred foods. However, when food deprivation was combined with a Premack principle-based treatment where participants received access to bites of preferred food contingent upon acceptance of non-preferred foods, acceptances increased. Levin and Carr reported that the intervention was not effective if participants were allowed free access to preferred foods for 2 hours preceding the experimental session. 
Although little research has been conducted on the effects of contextual stimuli on mealtime problem behavior, a relatively large body of research has focused on the relation between contextual or contextual stimuli and problem behavior occurring outside of mealtime. For example, Carr, Yarbrough, and Langdon (1997) assessed the effects of contextual stimuli on the occurrence of problem behavior emitted by 3 individuals diagnosed with autism. Functional analyses were conducted with participants to identify those variables responsible for the maintenance of problem behavior. These analyses were inconclusive as little or no problem behavior was observed. Idiosyncratic contextual stimuli (e.g., requests to complete puzzles) hypothesized to be related to problem behavior were identified for each participant by observing participants in the natural environment and interviewing caregivers. When these idiosyncratic variables were included in the functional analyses, clear functional relations were identified for all participants. In a study focusing specifically on escape maintained self-injurious behavior (SIB) emitted by nine individuals with developmental disabilities, Smith, Iwata, Goh, \& Shore (1995) demonstrated that the frequency of SIB was differentially related to specific characteristics of the requested task including duration of prompting, rate of presentation, and task novelty.

Several studies have shown that inclusion of idiosyncratic variables in a pre-treatment functional assessment leads to identification of more specific hypotheses about functional relations and hence, efficacious interventions (e.g., English \& Anderson, 2004). For example, Horner, Day, and Day (1997) assessed the influence of establishing operations (a variable that influences the reinforcing effectiveness of a given reinforcer) and contextual stimuli on problem behavior exhibited by three adolescents with developmental disabilities. Results of functional analyses suggested that the problem behavior of each participant was maintained by either 
escape from demand situations or access to preferred tangible items. After completion of the functional analysis, caregivers were interviewed to identify contextual events that might alter the function of reinforcing consequences (i.e., potential establishing operations, for example a cancelled visit from a parent or sleeping less than 5 hours per night). Horner et al. then systematically manipulated the putative establishing operations while holding the contextuals and consequences (e.g., presentation of requests, escape from task) constant. All participants emitted problem behavior after the manipulated establishing operations and little or no problem behavior when the establishing operations were not implemented. Interventions designed to alter the effects of the idiosyncratic contextual stimuli effectively reduced problem behavior for all participants.

Statement of the Problem

Taken together, the results of existing studies suggest that contextual variables might differentially influence mealtime behavior, but more research is needed. As noted earlier, escape extinction is one of the most commonly used interventions for food selectivity. Although escape extinction has been demonstrated to be effective in inpatient settings with trained therapists (e.g., Ahearn et al., 1996; Babbitt et al., 1994; Cooper et al., 1995; Didden et al., 1999; Hoch et al., 1994), it often is difficult to implement at the outset, leading some researchers to suggest that caregivers may be unable or unwilling to implement the intervention until significant gains are made or if trained therapists are not directly available to assist (Babbitt, Hoch, Sestero \& Cataldo, 1991). In fact, anecdotal observations from our laboratory suggest that caregivers often are hesitant or unwilling to implement the intervention without a great deal of support. If the presence or absence of specific contextual stimuli decreases the occurrence of mealtime behavior problems or increases acceptance of less preferred foods, then those stimuli might be 
manipulated to decrease the occurrence of mealtime behavior problems that parents find aversive (e.g., screaming, disruptive behavior, prolonged latency to acceptance) during the implementation of escape extinction. This may make it more likely that caregivers will use the intervention. Further, the overall outcome of escape extinction may be enhanced if manipulating contextual variables result in desired outcomes such as more rapid increases in the amount or variety of foods accepted or a greater reduction in the occurrence of disruptive behavior.

The purpose of the present investigation was to further evaluate the relation between contextual variables and mealtime behavior problems in the natural environment. In the first phase, two assessments were conducted. The first, a food preference assessment was conducted to identify preferred and non-preferred food items. The second, a pre-feeding assessment was conducted to determine the average amount of a preferred food a child would consume when given free access to that food. Variables commonly associated with mealtime (i.e., access to preferred tangibles, the presence of family members, presence of both non-preferred and preferred foods, and level of food deprivation) were systematically manipulated to examine their affect on mealtime behaviors during Phase 2.

Phase 1: Preference and Pre-Feeding Assessments

Method

\section{Participants and Setting}

Four children who displayed food selectivity and their mothers served as participants in this study. Participants were recruited through referrals made to the West Virginia University Center for Excellence in Disabilities Feeding and Swallowing Clinic. Prior to participation in the study, an interdisciplinary evaluation was conducted to rule out physical or physiological causes (e.g., dysphasia, reflux) for mealtime difficulties. Only children whose mealtime behavior 
problems were not directly related to an ongoing organic or structural problem participated in the study. At the clinic, a structured interview (standard part of the clinic visit) was administered by a graduate student on practicum at the clinic to develop hypotheses about environmental variables related to mealtime problem behavior.

Greg was a 4-year-old boy diagnosed with autism who engaged in aggression, SIB, interruptions, negative vocalizations, and crying during mealtimes. Maggie was a 2-year-old girl diagnosed with Down Syndrome and developmental delay. She exhibited aggression, selfinjurious behavior (SIB), interruptions, and crying during mealtimes. Sally was a 9-year-old girl diagnosed with autism. She exhibited aggression, SIB, interruptions and disrobing during mealtimes as well as at other times throughout the day. Sean was a 2-year-old boy diagnosed with Down Syndrome who exhibited aggression, SIB, interruptions, and crying during mealtimes.

The study was conducted in the homes of participant dyads (i.e., the caregiver and child's home) for 3 of the 4 participants. For Sally, the preference assessment was conducted in the clinic and the pre-feeding assessment was conducted at home. The preference assessment was conducted in the clinic as Sally exhibited high rates of aggressive behavior when asked to consume non-preferred foods and also often left the meal setting. Thus her parents were concerned about their safety and about containing Sally in the treatment area if the experiment was conducted at home.

Response Measurement and Interobserver Agreement

All meals were videotaped for later coding. All observations were recorded on laptop or desktop computers using the Observer (Noldus Information Technology) data-compilation 
program. This program allows for real-time recording of environmental events and responses using frequency, interval, or duration recording.

Frequency data were collected on five child responses: acceptances, scored when the child opened his or her mouth (e.g., to accept the bite, to scream) such that food could be placed in the mouth; expulsions, recorded when a bite of food larger than a pea appeared outside of the perimeter of the lips; disrobing, (Sally) scored when she had completely removed an article of clothing; negative vocalizations, (Greg) scored when a child verbally indicated displeasure (e.g., stated, “No!” or "Stop!”; and indication of preference for a food item, defined as the child looking at, reaching for, leaning toward, or verbally requesting the item (as described in the preference assessment below). Interruptions and problem behavior, defined as blocking presentation of bites (e.g., head turning, pushing spoon away with hands, attempting to get out of the seat), head-banging (Greg, Maggie), banging hand on table (Maggie), hand biting (Sally); hitting (Greg, Maggie, Sally, and Sean) kicking, pinching, and biting (Sally); were scored as either occurring or not occurring in continuous 5-s intervals during which a bite was presented. If a child did not accept a bite and simply sat still with the mouth clamped shut without turning the head or blocking the mouth, interruptions were not scored. Finally, crying was recorded as a duration measure. There was a 3-s delay prior to coding the onset and offset of crying such that the behavior was scored as occurring after the child had cried for 3 consecutive seconds and, after the child had discontinued crying for $3 \mathrm{~s}$, the behavior was coded as having ceased.

Caregiver responses were recorded during continuous 5-s intervals using a partial-interval recording procedure. Coded responses included prompts, delivery of attention or preferred tangible items, labeling of food items, and spoon removal. Three types of prompts were coded: specific prompts, defined as clear and distinct verbal statements directly related to taking a bite 
(e.g., “take a bite”, “open your mouth”, and “chew it up”) or statements offering a reward following the occurrence of a specified behavior (e.g., "eat the fruit and you can watch television”); spoon prompts, defined as holding the spoon within approximately $8 \mathrm{~cm}$ of the child's mouth; and vague prompts, defined as suggestive statements as opposed to direct statements, (e.g., “let’s eat”) and questions (e.g., “do you want some carrots?”). Caregiver delivery of attention or tangible items were defined as verbally praising a child's eating (e.g., “good boy”, "way to go”), physical attention such as patting on the back or hugging, and allowing access to tangible items such as preferred toys, preferred food, or a videotape. Data were collected as well on the type of food offered to ensure that caregivers offered targeted foods during the assessment. Whether the caregiver correctly verbally labeled the foods when presented (as in the preference assessment, described below) and removed foods (or spoon) from the child's mouth were also collected.

Interobserver agreement was assessed by trained observers who simultaneously but independently collected data during at least $30 \%$ of sessions for each participant dyad. Prior to initiating data collection, observers were trained using videotapes from prior feeding studies. Interobserver agreement scores were $80 \%$ or greater on all responses for three consecutive sessions prior to initiating data collection. If, after commencement of data collection, agreement scores fell below $80 \%$ on any target response for three consecutive sessions, data collection would have been suspended and observers would have been retrained in data collection procedures to pre-collection standards. However, retraining was not necessary as trained observers never fell below $80 \%$ agreement on any behavior.

For child responses coded as frequency measures, agreement was calculated by dividing the session into continuous 5-s intervals and comparing the observers' records for each interval. 
For each interval, the smaller number of recorded responses was divided by the larger number of recorded responses. The proportions then were averaged across the session and multiplied by 100 to obtain an agreement coefficient. Agreement coefficients for acceptances were 100\% (range 99-100\%), 100\% (range 99-100\%), 100\% (range 98-100\%), and 100\% (range 97-100\%) across Greg, Maggie, Sally, and Sean respectively. Agreement coefficients for expulsions were 100\% (range 97-100\%), 100\% (range 98-100\%), 100\% (range 98-100\%), and 100\% (range 99-100\%) across Greg, Maggie, Sally and Sean respectively. Agreement coefficients for aggression were 86\% (range 82-94\%), 87\% (range 85-90\%), and 83\% (range 79-90\%) across Greg, Maggie and Sally respectively. Agreement coefficients for SIB were 100\% (range 99-100\%), 90 (range 8596\%), and 100\% (range 98-100) across Greg, Maggie and Sally respectively. Agreement coefficients for indication of preference were 100\% (all were 100\%), 100\% (range 99-100\%), 100\% (range 98-100\%), and 100\% (range 98-100\%) across Greg, Maggie, Sally and Sean. The agreement coefficient for negative vocalizations (Greg) was 94\% (range 90-99\%). The agreement coefficient for disrobing (Sally) was 100\% (all were 100\%)

Total agreement coefficients were calculated for child interruptions, crying and all caregiver responses. Total agreement was calculated by dividing the number of intervals in which observers agreed on either the occurrence or nonoccurrence of the response divided by the total number of intervals. To obtain an agreement coefficient, the result of each formula was multiplied by 100. Mean interobserver agreement across participants was 83.3\% (range 76-95\%) for interruptions and $100 \%$ range (99-100\%) for crying.

The results of total agreement calculations for each caregiver behavior were averaged between the preference and pre-feeding assessments. Mean interobserver agreement across participants was 93\% (range $86-100 \%$ ) for specific prompts, 100\% (all were 100\%) for vague 
prompts $100 \%$ (all were 100\%) for initial spoon prompts, 100\% (all were 100\%) for continued spoon prompts 96.5\% (range 88-100\%) for correct labeling of foods, 98.3\% (range 95-100\%) for spoon removal, 100\% (all were100\%) for delivery of attention, and 100\% (all were 100\%) for delivery of preferred tangible items.

\section{Procedure}

Caregivers were trained to conduct all phases of the investigation. After a general overview, caregivers were trained prior to each phase. Training consisted of the provision of written materials and viewing videotaped role-plays created by graduate and undergraduate students depicting the experimental arrangements. In addition, caregivers rehearsed with the primary investigator acting as the child until the caregiver responded appropriately at least $90 \%$ of the time during three consecutive rehearsal sessions. Conditional probabilities were calculated to evaluate treatment integrity (see Data Analysis section). Integrity data continued to be collected throughout the study. If any response had been implemented incorrectly more than 3 times consecutively, then procedures would have ceased until the caregiver was retrained to criteria. This never occurred. After each session the experimenter provided immediate feedback regarding implementation of procedures.

Food preference assessment. A forced-choice preference assessment (described below) similar to that used by Fisher et al., (1992) was conducted to determine a hierarchical food preference for the 3 participants who were not self-feeders (Greg, Maggie, and Sean). Sally's food preference assessment was conducted using a free-operant paradigm across three consecutive sessions. That is, 2 preferred and 3 non-preferred foods were concurrently available to Sally from which she could chose from and self-feed across three consecutive sessions. Foods evaluated in the preference assessment included two reportedly preferred and three reportedly 
non-preferred foods as identified during the caregiver interview. Non-preferred foods included food items that the child either refused to consume or accepted only after emitting behavior problems. Preferred foods included items that the child purportedly consumed willingly, with little or no prompting or without emitting problem behavior.

During the forced-choice preference assessment caregivers presented food items in pairs such that each food was paired with every other food, with each pair being presented twice in a counterbalanced order. Each trial began with the presentation of a food pair (e.g., a bite of yogurt and a bite of applesauce) placed on a plate approximately $15 \mathrm{~cm}$ apart and $20 \mathrm{~cm}$ in front of the child. The location of the bite on the plate (e.g., left side) was counterbalanced across the two presentations. To help the child distinguish between foods, the caregiver held up and verbally labeled each food item prior to presentation. If the child approached one of the foods (by verbally requesting the item, grasping the item and placing it in the mouth, reaching for the item, or leaning towards the item), the other food was removed. If the child did not pick the item up, the caregiver held the bite at the child's lips for $5 \mathrm{~s}$ or until the child opened his or her mouth such that the bite could be placed in the mouth. If neither food was selected within $5 \mathrm{~s}$ the trial was scored as "no response." If the child interrupted the presentation of bites or emitted disruptive behavior caregivers were instructed to ignore both types of behavior.

Three concurrent free-operant assessment sessions were conducted with Sally. At the beginning of each session, 5 food items were placed in front of her and Sally's mother instructed Sally to take a bite approximately every 30 seconds during a 5-min to 10 -min session. Sessions were terminated after Sally had not taken a bite of any food for one min.

For each child, foods accepted on $80 \%$ of the presentations were considered preferred food items, and foods accepted during less than $20 \%$ of the presentations were considered non- 
preferred foods. Identified non-preferred foods were designated as target foods used throughout the remainder of the experiment.

Pre-feeding assessment. After completion of the food preference assessment, a prefeeding assessment was conducted. The purpose of this assessment was to determine the average amount of preferred foods the child would consume, given free access. The assessment was conducted after the child had not consumed any foods for at least 4 hours and no liquids for at least 1 hour (by parent report). During the assessment, Greg and Sally were given unlimited access to the preferred food identified in the preference assessment. Maggie's and Sean's caregivers were concerned about allowing free access to preferred foods as children with Down Syndrome often gain weight easily. Thus, for both participants the pre-feeding assessment was modified as follows. First, children were not allowed free access to food, but rather were presented with only as much of the preferred food as would typically be offered at meal time, given that the meal consisted only of that food. For example, Sean was offered 5.8 oz of baby food bananas and Maggie was offered $5.3 \mathrm{oz}$ of baby food wild blueberries.

For Greg, Maggie, and Sean who did not self-feed, the parent held a spoonful of food in front of the child. If the child opened his or her mouth, the caregiver placed the bite of food in the child's mouth. There were no programmed consequences for acceptances, expulsions, or interruptions. Bites were presented on a fixed-time 10 s schedule (FT $10 \mathrm{~s}$ ). The meal was terminated after the child refused 4 consecutive bites of food. For Sally, who fed herself, the food was placed on a plate in front of her and her mother stated, "It is time to eat, eat as much as you want." If Sally failed to take a bite for 5 consecutive min, the parent asked, "Is that all you want?” If Sally did not take a bite for 6 consecutive mins, the meal was terminated. 
The pre-feeding assessment was conducted at least three times on separate days. If, after 3 meals were conducted and the amount consumed in each meal was within $10 \%$ of the mean for those meals, the assessment was terminated. If the amount was not within $10 \%$ of the mean for those meals, the assessment was conducted until three consecutive meals were within $10 \%$ of the mean of those meals or until 10 meals were conducted. If the amount consumed per meal had continued to vary widely after 10 meals, the first author would have met with her chair to determine a course of action. However, it was not necessary to conduct more than 5 meals for any participant in this study.

\section{Data Analysis}

To evaluate the extent to which assessments were implemented with procedural integrity, conditional probabilities were calculated throughout the study. Proportions that were calculated for the preference assessment are depicted in Table 1. For most proportions, two formulas were used, the first formula was used to measure the proportion of parent behaviors that followed a relevant child behavior (parent-based calculations); for example, the proportion of spoon prompts that followed an indication of preference. The second formula was used to measure the proportion of child behavior that preceded a relevant parent behavior (child-based calculations); for example, the proportion of times the child indicated a preference that was followed by a spoon prompt. For child behaviors, all probabilities were calculated based on the first occurrence of a child behavior within each interval (i.e., as though child behavior was coded as partialinterval measure). This allowed for an interval-by-interval comparison of both parent and child data.

Results and Discussion

Preference Assessment 
Results of the preference assessment for all participants are presented as the proportion of accepted bites not expelled in Figure 1. Preferred foods are labeled in all capital letters on the left-most side of the figure. Non-preferred foods presented to Greg during the preference assessment included sweet peas and peaches and preferred foods were toast and chocolate chip cookies. Greg accepted and swallowed all presentations of chocolate chip cookies (upper panel, Figure 1) but refused all other foods including the reportedly preferred toast. Chocolate chip cookies were used during the pre-feeding assessment.

Reportedly non-preferred foods presented to Maggie during the preference assessment included pasta with spaghetti sauce, green beans and potatoes; reportedly preferred foods offered were pear and wild blueberry pureed baby foods. The proportion of accepted bites not expelled (i.e., bites swallowed) are depicted in the second panel of Figure 1. Maggie accepted the reportedly preferred foods each time they were offered and never accepted any of the purported non-preferred foods. Thus, blueberries were used for the pre-feeding assessment.

Reportedly non-preferred foods presented to Sally were salad with dressing, green beans, and applesauce. Preferred foods included mashed potatoes with Ranch dressing and a peanut butter sandwich. Sally only ate the mashed potatoes with Ranch dressing and did not eat the other food items (third panel, Figure 1).

Reported non-preferred foods presented to Sean during the preference assessment included potatoes, diced chicken, and diced apples. Preferred foods offered were banana stage-2 baby food and tender sweet peas. Results of the preference assessment with Sean are in the bottom panel of Figure 1 . Sean consumed $83 \%$ of bites offered of bananas and $42 \%$ of bites offered of sweet peas. He never consumed more than $20 \%$ of offered bites of non-preferred foods and never consumed any bites of apples. Bananas were used during the pre-feeding assessment. 


\section{Pre-Feeding Assessment}

Three pre-feeding meals consisting of unlimited access to chocolate chip cookies were conducted with Greg. During each meal Greg consumed an average of 2.25 oz. or about 2 chocolate chip cookies. Although this seems like a rather small amount of food, Greg's mother reported that Greg rarely consumes very much food at one setting. During the modified prefeeding assessment, Maggie consumed 100\% of available baby food wild blueberries (5.3 ounces at each of the three meals). Sean consumed $100 \%$ of available baby food bananas at each of the tree meals (5.8 ounces per meal). Three pre-feeding meals consisting of unlimited access to mashed potatoes were conducted with Sally and the average amount consumed during the meals was $6.5 \mathrm{oz}$.

Results of integrity calculations for each participant are depicted in Table 2. The formulas used to derive these proportions are displayed in Table 1. All caregivers correctly labeled foods, presented prompts, and removed the spoon in accordance with experimental procedures $100 \%$ of the time.

Phase 2: Contextual Variable Evaluation

\section{Method}

\section{Participants and Setting}

The participants were the same as those in experiment 1. For all participants, except Sally, Experiment 2 was conducted in the dyad's homes. For Sally, Experiment 2 was conducted in the clinic.

Response Measurement and Interobserver Agreement

Again, all meals were videotaped for later coding. Target responses and operational definitions were identical to those used in Experiment 1. Agreement coefficients for frequency- 
scored child behaviors, interval-scored child behaviors (interruptions and crying) and parent behaviors were computed as described in Experiment 1.

Agreement coefficients for acceptances were 100\% (range 99-100\%), and 100\% (range 99-100\%), across Sally and Sean respectively. Agreement coefficients for expulsions were 100\% (range 99-100\%) and 100\% (range 98-100\%) for Sally and Sean respectively. Agreement coefficients for aggression were 95\% (range 92-99\%), 79\% (range 70-89\%), and 84\% (range 80-90\%) across Greg, Maggie and Sally. Agreement coefficients for SIB were 83\% (range 8288\%), 67\% (range 65-83\%), and 94\% (range 90-100\%) across Greg, Maggie and Sally. Agreement coefficients for indication of preference were 100\% (all were 100\%) for each child. The agreement coefficient for negative vocalizations was $80 \%$ (range $75-88 \%$ ) for Greg. The agreement coefficient for disrobing was 100\% (all were 100\%) for Sally. For partial-interval data the mean interobserver agreement across participants was 83.3\% (range 76-95\%) for interruptions and 100\% (range 99-100\%) for crying.

The mean interobserver agreement across participants for caregiver behaviors was $90 \%$ (range $86-93 \%$ ) for specific prompts, 100\% (all were 100\%) for vague prompts, 92\% (range 9193\%) for initial spoon prompts, 100\% (all were 100\%) for continued spoon prompts, $92 \%$ (range 89-96\%) for correct labeling of foods, 91\% (range 82-100\%) for spoon removal, 100\% (all were $100 \%$ ) for delivery of attention, and $100 \%$ (all were $100 \%$ ) for delivery of preferred tangible items.

Procedure

Caregiver training. Training of caregivers consisted of the provision of written materials and viewing videotaped role-plays created by graduate and undergraduate students depicting the experimental arrangements. In addition, caregivers rehearsed with the primary investigator acting 
as the child until the caregiver responded appropriately at least $90 \%$ of the time during three consecutive rehearsal sessions. Conditional probabilities were calculated to evaluate treatment integrity (see data analysis section). Integrity data continued to be collected throughout the study. If any response had been implemented incorrectly more than 3 times consecutively, then procedures would have ceased until the caregiver was retrained to criteria; this was not necessary for any caregiver during implementation of experimental procedures.

Contextual variable evaluation. Two multielement manipulations were used to evaluate the effects of contextual variables on the occurrence of expulsions, interruptions, problem behavior, crying, and percentage of bites accepted. These variables were examined under conditions of food deprivation and session pre-feeding, the order of which was counterbalanced across participants. The second set of contextual variables consisted of the presence or absence of the following: tangible, preferred-food, and idiosyncratic stimuli. These variables were manipulated systematically within the presence of food deprivation and satiation. Thus, the design consisted of two multielement designs (second set of contextual variables) nested within an $\mathrm{AB}$ design (food deprivation and session pre-feeding).

Sessions were conducted at about the time the child typically ate. Two to ten sessions were conducted per day; the mean number of sessions conducted per day was 7.6. Across conditions, a session consisted of the presentation of 10 bites of each of the two non-preferred food items identified in the preference assessment of Experiment 1. The order of presentations of foods was alternated within each session. At least three sessions were conducted per condition and sessions were conducted until a trend was observed. For each presentation, a bolus of food was presented on a spoon held within approximately $3 \mathrm{~cm}$ of the child's lips with the verbal prompt, “take a bite.” Each bite was held at the child’s lips for 5 s or until an acceptance 
occurred. There were no programmed consequences for non-acceptances, expulsions, interruptions, or acceptances of bites presented. The next scheduled bite was presented $5 \mathrm{~s}$ after the spoon was removed. For example, if the child did not accept the bite within $5 \mathrm{~s}$, the spoon was removed and the next bite was presented $5 \mathrm{~s}$ later. If the bite was expelled the caregiver presented the next bite $5 \mathrm{~s}$ after the expulsion. If the bite was accepted, the spoon was removed and the next presentation was delivered $5 \mathrm{~s}$ after the acceptance.

In the tangible condition, a preferred tangible item (identified by caregiver in preexperimental interview) was present throughout the session. For example, if the preferred tangible item was a doll, the doll was made available to the child throughout the meal. In the family-present condition, family members that typically ate with the child were present. Individuals were instructed to interact with the child as they normally would during meals while the target caregiver implemented the bite presentations as described above. Behaviors or prompts emitted by individuals present during this meal were recorded separate from those delivered by the primary feeder (caregiver). In the preferred-food condition, a preferred food (as determined by the preference assessment) was placed on the plate next to the target foods. If the child requested or moved toward the preferred food the caregiver was instructed to tell the child he or she could have it later. The idiosyncratic-variable condition was conducted if, during the preexperiment interview, caregivers identified other variables (e.g., using a specific plate, when a particular television show was on) they believed influenced mealtime behavior. In this condition the variable identified by caregivers was present throughout the session. Finally, in the control condition, the prompting procedure described above was used. In the control condition, family members, preferred foods, tangible items and idiosyncratic variables were not present. 
To evaluate the effect of relative food deprivation on other contextual variable manipulations, each contextual condition (described above) was conducted at least 3 times when the child was food deprived and 3 times when the child participant was pre-fed at the time the session was conducted. The order of presentation of sessions of food deprivation and session prefeeding were counterbalanced across participants. A child was considered food deprived if he or she had not consumed any food within 4 hours of the session and had consumed no more than 5 ounces of liquid within 1 hour of the session. Prior to pre-feeding sessions, parents were asked to restrict access to foods for at least 4 hours. Thirty min prior to the onset of the contextual variable evaluation, the parent was instructed to allow the child unlimited access to preferred food identified in the preference assessment. If the child consumed $90 \%$ or more of the average amount of preferred food consumed in the pre-feeding assessment, then pre-feeding meals commenced. If after 3 sessions, an observable difference between pre feeding and deprivation was not noted, further sessions were conducted. Six sessions of each condition were conducted with Maggie and Sean, and 3 sessions per condition were conducted with Greg and Sally.

\section{Data Analysis}

To evaluate the extent to which procedures were implemented with integrity, conditional probabilities were calculated. Proportions that were calculated for the evaluation are depicted in Table 3. Again, two formulas were calculated, parent-based and child-based calculations. For child behaviors, all probabilities were calculated based on the first occurrence of a child behavior within each interval (i.e., as though child behavior was coded as partial-interval measure). This allowed for an interval-by-interval comparison of both parent and child data.

Results and Discussion

Contextual Variable Evaluation 
Greg. Results obtained with Greg are depicted in Figures 2 and 3. Bar graphs in Figure 2 are means of all sessions for each variable condition arrangement. These data are presented as line graphs in Figure 3. On line graphs of each child's data the long tick marks on the x-axes divide sessions that were conducted on the same day. A total of eight different arrangements of contextual variable conditions were conducted with Greg: preferred food present (chocolate chip cookies), tangible (toy car), and idiosyncratic (preferred videotape), and a control condition under first the condition of session pre-feeding and subsequently food deprivation. A total of 3 sessions were conducted for each of the eight variable combinations.

Greg did not accept and swallow non-preferred foods in any condition of the assessment (top panel, Figure 2). The mean proportion of intervals scored with interruptions and other problem behavior (second panel, Figure 2) and crying (third panel, Figure 2) occurred less often in the presence of the idiosyncratic stimulus under pre-feeding. In fact, crying was never observed in the pre-feeding condition in the presence of the idiosyncratic stimulus. The bottom panel (Figure 2) shows mean negative vocalizations per minute. Negative vocalizations occurred least often in the presence of the tangible item in the pre-feeding condition. However, because responding decreased systematically across pre-feeding sessions, this finding should be interpreted with caution (bottom panel, Figure 3). Under conditions of deprivation, negative vocalizations occurred only rarely.

In summary, data collected with Greg indicate that, in the context of pre-feeding, the presence of the idiosyncratic stimulus resulted in reductions in interruptions and crying. Deprivation may have decreased negative vocalizations (again, this should be interpreted with caution due to the decreasing trend across session) but otherwise had little to no effect on Greg's mealtime behavior. 
Maggie. Results obtained with Maggie are depicted in Figures 4 and 5. Bar graphs in Figure 4 are mean calculations of all sessions for each variable condition arrangement. In addition, these data are presented as line graphs in Figure 5. A total of eight different arrangements of contextual variable conditions were conducted with Maggie. These consisted of preferred food present (wild blueberry baby food), tangible (toy frog doll on highchair tray), idiosyncratic (preferred videotape), and control conditions under pre-feeding and subsequently food deprivation conditions. Six sessions were conducted for each of the eight variable combinations.

The upper panel of Figure 4 shows mean accepted bites not expelled per minute for Maggie. Maggie never accepted a bite of the non-preferred foods during the assessment. The middle panel of Figure 4 shows the mean proportion of intervals scored with interruptions and other behavior. Response rates increased over the course of the pre-feeding phase and remained elevated throughout the deprivation phase (upper-right panel, Figure 5). In addition, there were fewer interruptions under session pre-feeding and in the idiosyncratic condition. Crying was observed in all conditions except the idiosyncratic condition under both pre-feeding and deprivation (bottom panel, Figure 4). Crying also occurred only rarely in the presence of a preferred tangible (toy frog) during pre-feeding.

In summary, the presence of the idiosyncratic stimulus (preferred video) resulted in suppression of crying and across both pre-feeding and deprivation conditions. Further, relatively less crying was observed in the presence of a preferred tangible item during session pre-feeding (presence of toy frog on highchair tray). Also, interruptions were the lowest during the idiosyncratic condition under the condition of session pre-feeding. 
Sally. Results obtained with Sally are depicted in Figures 6 and 7. Bar graphs in Figure 6 are mean calculations for all sessions of each variable condition arrangement. These data are also presented as line graphs in Figure 7. A total of ten different arrangements of contextual variable conditions were conducted with Sally which consisted of a preferred-food present (e.g., mashed potatoes with Ranch dressing), tangible (e.g., Leap Frog Learning Pad), idiosyncratic (e.g., preferred videotape), family present (e.g., father in the room) and control conditions under first the condition of food deprivation and subsequently session pre-feeding. A total of 3 sessions were conducted for each of the ten variable combinations.

Sally typically "tasted" food by licking a small amount off the spoon, rather than swallowing an entire bolus. Thus, the upper panel of Figure 6 reflects the number of tastes of bites tasted not expelled per minute that Sally took of food items. Overall, Sally tasted more bites of the presented non-preferred foods under conditions of session pre-feeding, however, bites tasted were extremely variable (upper left panel, Figure 7). In the presence of a family member, bites tasted were roughly equivalent across the two phases. Similar patterns were observed for interruptions and other behavior (second panel, Figure 6), which occurred less often in the presence of pre-feeding, and were rarely observed in the presence of the idiosyncratic stimulus in pre-feeding sessions. Crying rarely occurred during the assessment and, with the exception of one session in pre-feeding, never occurred during the pre-feeding (third panel, Figure 6).

Disrobing (bottom panel, Figure 6) occurred intermittently throughout the assessment under both deprivation and pre-feeding conditions. Disrobing never occurred during the idiosyncratic, family-present, and control conditions under conditions of food deprivation. In addition, disrobing did not occur during the idiosyncratic and tangible conditions under the condition of session pre-feeding. 
In summary, no particular condition consistently and significantly reduced the emission of problem behavior, interruptions, or disrobing. However, it should be noted the overall number of tastes of food was greatest under the condition of session pre-feeding and that the proportion of intervals scored with interruptions and crying were both reduced under conditions of session pre-feeding.

Sean. Results obtained with Sean are depicted in Figures 8 and 9. Bar graphs in Figure 8 are mean calculations for all sessions of each variable condition arrangement. These data are also presented as line graphs in Figure 9. A total of eight different arrangements of contextual variable conditions were conducted with Sean including preferred food present (pureed banana baby food), tangible (blue bowl), idiosyncratic (preferred videotape), and subsequent control condition under food deprivation and subsequently session pre-feeding conditions. A total of six sessions were conducted for each of the eight variable combinations.

Sean occasionally accepted and swallowed bites of non-preferred food under conditions of deprivation in the preferred food (pureed banana baby food) present condition (upper panel, Figure 8). In the pre-feeding condition, he occasionally accepted and swallowed bites in the control condition, preferred-food present condition and in the idiosyncratic condition (videotape). Interruptions and other behavior occurred less often in the presence of the idiosyncratic variable (middle panel, Figure 8) across both deprivation and pre-feeding. The bottom panel of Figure 8 illustrates the proportion of intervals scored with crying. Crying was observed during only one session of the idiosyncratic condition in the presence of deprivation and crying never occurred during this condition in the presence of pre-feeding. 
In summary, in the presence of the idiosyncratic variable (preferred videotape), the proportion of intervals scored with interruptions and crying was greatly reduced relative to other contextual variable arrangements.

Again, conditional probabilities were calculated throughout the study to assess treatment integrity. Proportions that were calculated for the contextual variable evaluation are depicted in Table 4 and the formulas from which proportions were derived are in Table 3. For each child the proportion of specific prompts delivered after the start of trial was .99 and the proportion of initial spoon prompts delivered after the start of a trial was .99, indicating that prompts were delivered accurately. In addition, for each child the proportion of attention deliveries following interrupted or expelled bites of food was .00; as attention was never delivered following such responses.

\section{General Discussion}

Taken together, the results of this study indicate that manipulation of contextual variables may affect the occurrence of mealtime problem behavior. Interestingly, consistent results were not observed across or within participants. Rather, contextual variables had rather specific and individualized effects on the responding emitted by participants.

One variable that had a more generalized effect was that in the presence of the idiosyncratic stimulus three of four participants (Greg, Maggie, and Sean), emitted less interruptions during the idiosyncratic condition (preferred video) in the presence of session prefeeding (pre-feeding and deprivation for Sean). Similar effects (conceptualized as distraction) routinely are reported in the pediatric psychology literature. For example, research shows that noncontingent access to items such as books, video games, cartoons, and movies, reduces responses collectively labeled distress (e.g., crying, elevated heart rate) emitted by children 
during immunizations (e.g., Cohen, 2002; Manimala, Blount, \& Cohen, 2000; Cohen, Blount, Panopoulos, 1997) and dental procedures (e.g., Stark, et al., 1989).

These findings might be understood by examining the literature on response-independent stimulus delivery. Research conducted on problem behavior that occurs outside mealtime has shown that noncontingent access to reportedly preferred stimuli (e.g., attention, access to snacks, toy) often results in suppression of problem behavior (e.g., Lindberg, Iwata, Roscoe, Worsdell, \& Hanley, 2003; Ringdahl, Winborn, Andelman, \& Kitsukawa, 2002; Fisher, O'Connor, Kurtz, DeLeon, \& Gotjen, 2000; Hagopian, Crockett, van Stone, DeLeon, \& Bowman, 2000; Hanley, Piazza, \& Fisher, 1997; Fischer, Iwata, \& Mazaleski, 1997). In the majority of the above-cited studies, engagement with the delivered stimulus required some sort of consummatory response that often was incompatible with engagement in problem behavior (e.g., the child could not interact with a toy and use the hands to engage in SIB), which in the present investigation was not necessarily the case. That is, children in the present investigation could watch a video tape and still hit the spoon, cry, or engage in SIB. It is possible that emitting such responses decreased interaction with the tangible item and when emitting problem behavior, the child could not see or hear the video as well.

An additional finding, that two of the four participants (Greg and Sally) exhibited decreased problem behavior only under conditions of session pre-feeding was somewhat surprising. To date only one published applied study has examined the role of session prefeeding. Levin and Carr (2001) demonstrated that acceptance of non-preferred food items only occurred when prior access to preferred foods was limited and a Premack principle based intervention was used concurrently. Problem behavior was reduced during this condition arrangement; however, they did not report differential findings of pre-feeding on the occurrence 
of interruptions. Typically therapists request that food selective individuals be food deprived at the beginning of treatment sessions, and add that this is suggested to increase the likelihood that the she will be hungry and be more inclined to accept bites of non-preferred foods when presented to her. However, this notion of increasing the acceptance of non-preferred foods when presented was not confirmed by this study. Further, findings indicate that if anything session prefeeding may be suggested for some individuals in order to reduce problem behavior emitted by the individual during treatment sessions. If parents are conducting escape extinction in their home this reduction in mealtime problem behavior may make it much more likely that they will continue to use the intervention to increase the amount and variety of foods that her child consumes.

There are several limitations in the current investigation. First, it is possible that if more sessions had been conducted with Greg and Sally that the effect of session pre-feeding in the reduction of mealtime problem behavior would have been diminished. An additional limitation is that two of the participants (Maggie and Sean) were not given completely free access to preferred food items in the pre-feeding assessment or during the pre-feeding conditions of Experiment 2. However, it should be noted that when the other two participants (Greg and Sally) were given free access to preferred foods their mothers stated that they had consumed the “typical” amount they would during a meal situation.

One additional limitation in the current investigation is the variability in the data. One explanation for the variability observed is that because several sessions were conducted per day, child participants may have become fatigued and as a consequence interruptions and crying may have increased. This trend is most observable in the data obtained of the proportion of intervals 
scored with crying with Sean (bottom panel, Figure 9). Future research should evaluate whether extraneous variability may be reduced by conducting fewer sessions per day.

Variability also may be reduced by assessing the effects of fewer contextual variables in any one investigation. In the current study, it is possible that some of the variability may have been due to carryover across conditions. This might be reduced by conducting fewer conditions overall and by making efforts to enhance the discriminability of the conditions (e.g., running in different rooms). Future research should evaluate whether manipulation of contextual variables such as those investigated in the current study affects the outcome of interventions such as escape extinction. 


\section{References}

Ahearn, W. H., Kerwin, M. E., Eicher, P. S., Shantz, J., \& Swearingin, W. (1996). An alternating treatments comparison of two intensive interventions for food refusal. Journal of Applied Behavior Analysis, 29, 321-332.

Anderson, C. M., \& McMillan, K. (2002). Parental use of escape extinction and differential reinforcement to treat food selectivity. Journal of Applied Behavior Analysis, 34, 511515.

Babbitt, R. L., Hoch, T. A., Coe, D. A., \& Cataldo, M. F. (1994). Behavioral assessment and treatment of pediatric feeding disorders. Journal of Developmental and Behavioral Pediatrics, 15, 278-291.

Babbitt, R. L., Hoch, T. A., Sestero, D. G., \& Cataldo, M. F. (1991, May). Organizational and service evaluation of pediatric behavioral feeding program. Poster presented at the Convention of the Association for Behavior Analysis, Atlanta, GA.

Blount, R. L., Cohen, L. L., Frank, N. C., Bachanas, P. J., Smith, A. J., Manimala, M. R., et al. (1997). The Child-Adult Medical Procedure Interaction Scale-Revised: An assessment of validity. Journal of Pediatric Psychology, 22, 73-88.

Blount, R. L., Sturges, S. W., \& Powers, S. W. (1990). Analysis of child and adult behavioral variations by phase of medical procedure. Behavior Therapy, 21, 33-48.

Carr, E. G., Yarbrough, S. C., \& Langdon, N. A. (1997). Effects of idiosyncratic stimulus variables on functional analysis outcomes. Journal of Applied Behavior Analysis, 30, 673-686.

Cohen, L. L. (2002). Reducing infant immunization distress through distraction. Health Psychology, 21, 207-211. 
Cohen, L. L., Blount, R. L., Cohen, R. J., Schaen, E. R., \& Zaff, J. (1999). A comparative study of distraction versus topical anesthesia for pediatric pain management during immunizations. Health Psychology, 18, 591-598.

Cohen, L. L., Blount, R. L., and Panopoulos, G. (1997). Nurse coaching and cartoon distraction: An effective and practical intervention to reduce child, parent, and nurse distress during immunizations. Journal of Pediatric Psychology, 22, 355-370.

Cooper, L. J., Wacker, D. P., McComas, J. J., Brown, K., Peck, S. M., Richman, D., Drew, J., Frischmeyer, P., \& Millard, T. (1995). Use of component analyses to identify active variables in treatment packages for children with feeding disorders. Journal of Applied Behavior Analysis, 28, 139-153.

Didden, R., Seys, D., \& Schouwink, D. (1999). Treatment of chronic food refusal in a young developmentally disabled child. Behavioral Interventions, 14, 213-222.

Fischer, S. M., Iwata, B. A., \& Mazaleski, J. L. (1997). Noncontingent delivery of arbitrary reinforcers as treatment for self-injurious behavior. Journal of Applied Behavior Analysis, 30, 239-249.

Fisher, W., Piazza, C. C., Bowman, L., Hagopian, L. P., Owens, J. C., \& Sterns, I. (1992). A comparison of two approaches for identifying reinforcers for persons with severe and profound disabilities. Journal of Applied Behavior Analysis, 25, 491-498. 
Fisher, W. W., O'Connor, J. T., Kurtz, P. F., DeLeon, I. G., \& Gotjen, D. L. (2000). The effects of noncontingent delivery of high- and low-preference stimuli on attention-maintained destructive behavior. Journal of Applied Behavior Analysis, 33, 79-83.

Galensky, T. L., Miltenberger, R. G., Stricker, J. M., \& Garlinghouse, M. A. (2001). Functional assessment and treatment of mealtime behavior problems. Journal of Positive Behavior Interventions, 3, 211-224.

Hagopian, L. P., Crockett, J. L., van Stone, M., DeLeon, I. G., \& Bowman, L. G. (2000). Effects of noncontingent reinforcement on problem behavior and stimulus engagement: The role of satiation, extinction, and alternative reinforcement. Journal of Applied Behavior Analysis, 33, 433-449.

Hanley, G. P., Piazza, C. C., \& Fisher, W. W. (1997). Noncontingent presentation of attention and alternative stimuli in the treatment of attention-maintained destructive behavior. Journal of Applied Behavior Analysis, 30, 229-237.

Hoch, T. A., Babbitt, R. L., Coe, D. A., Krell, D. M., \& Hackbert, L. (1994). Escape extinction: Combining positive reinforcement and escape extinction procedures to treat persistent food refusal. Behavior Modification, 18, 106-128.

Horner, R. H., Day, H. M., \& Day, J. R. (1997). Using neutralizing routines to reduce problem behaviors. Journal of Applied Behavior Analysis, 30, 601-614.

Jay, S. M., Elliott, C. H., Fitzgibbons, I., Woody, P. \& Siegel, S. (1995). A comparative study of cognitive behavior therapy versus general anesthesia for painful medical procedures in children. Pain, 62, 3-9. 
Kedesdy, J. H., \& Budd, K. S. (Eds.). (1998). Childhood feeding disorders: Biobehavioral assessment and intervention. Baltimore, MD: Paul H. Brookes Publishing Company.

Kerwin, M. E., Ahearn, W. H., Eicher, P. S., \& Burd, D. M. (1995). The costs of eating: A behavioral economic analysis of food refusal. Journal of Applied Behavior Analysis, 28, $245-260$.

Kitfield, E. B., \& Masalsky, C. J. (2000). Negative reinforcement-based treatment to increase food intake. Behavior Modification, 24, 600-608.

Levin, L., \& Carr, E. G. (2001). Food selectivity and problem behavior in children with developmental disabilities: Analysis and intervention. Behavior Modification, 25, 443470.

Lindberg, J. S., Iwata, B. A., Roscoe, E. M., Worsdell, A. S., \& Hanley, G. P. (2003). Treatment efficacy of noncontingent reinforcement during brief and extended application. Journal of Applied Behavior Analysis, 36, 1-19.

Linscheid, T. R., Budd, K. S., \& Rasnake, L. K. (1995). Pediatric feeding disorders. In M. C. Roberts (Ed.), Handbook of pediatric psychology (pp. 501-515). New York, NY: Guilford Press.

Luiselli, J. K., Evans, T. P., \& Boyce, D. A. (1985). Contingency management of food selectivity and oppositional eating in a multiply handicapped child. Journal of Clinical Child Psychology, 14, 153-156.

Manimala, M. R., Blount, R. L., and Cohen, L. L. (2000). The effects of parental reassurance versus distraction on child distress and coping during immunizations. Children's Health Care, 29, 161-177. 
Mueller, M. M., Piazza, C. C., Moore, J. W., Kelley, M. E., Bethke, S. A., Pruett, A. E., Oberdorff, A. J., \& Layer, S. A. (2003). Training parents to implement pediatric feeding protocols, Journal of Applied Behavior Analysis, 36, 545-562.

Munk, D. D., \& Repp, A. C. (1994). Behavioral assessment of feeding problems of individuals with severe disabilities. Journal of Applied Behavior Analysis, 27, 241-250.

O’Reilly, M. F., \&Lancioni, G. E. (2001). Treating food refusal in a child with Williams syndrome using the parent as therapist in the home setting. Journal of Intellectual Disability Research, 45, 41-46.

Palmer, S., Thompson, R. J., \& Linscheid, T. R. (1975). Applied behavior analysis in the treatment of childhood feeding problems. Developmental Medicine and Child Neurology, 17, 333-339.

Patel, M. R., Piazza, C. C., Martinez, C. J., Volkert, V. M., \& Santana, C. M. (2002). An evaluation of two differential reinforcement procedures with escape extinction to treat food refusal. Journal of Applied Behavior Analysis, 35, 363-374.

Ringdahl, J. E., Winborn, L. C., Andelman, M. S., \& Kitsukawa, K. (2002). The effects of noncontingently available alternative stimuli on functional analysis outcomes. Journal of Applied Behavior Analysis, 35, 407-410.

Riordan, M. M., Iwata, B. A., Finney, J. W., Wohl, M. K., \& Stanley, A. E. (1984). Behavioral assessment and treatment of chronic food refusal in handicapped children. Journal of Applied Behavior Analysis, 17, 327-341.

Riordan, M. M., Iwata, B. A., Wohl, M. K., \& Finney, J. W. (1980). Behavioral treatment of food refusal and selectivity in developmentally disabled children. Applied Research in Mental Retardation, 1, 95-112. 
Shore, B. A., Babbitt, R. L., Williams, K. E., Coe, D. A., \& Snyder, A. (1998). Use of texture fading in the treatment of food selectivity. Journal of Applied Behavior Analysis, 31, $621-633$.

Smith, R. G., Iwata, B. A., Goh, H., \& Shore, B. A. (1995). Analysis of establishing operations for self-injury maintained by escape. Journal of Applied Behavior Analysis, 28, 515-535.

Stark, L. J., Allen, K. D., Hurst, M., Nash, D. A., Rigney, B. \& Stokes, T. F. (1989). Distraction: Its utilization and efficacy with children undergoing dental treatment. Journal of Applied Behavior Analysis, 22, 297-307.

Werle, M. A., Murphy, T. B., \& Budd, K. S. (1993). Treating chronic food refusal in young children: Home-based parent training. Journal of Applied Behavior Analysis, 26, 421433.

Werle, M. A., Murphy, T. B., \& Budd, K. S. (1998). Broadening the parameters of investigation in treating young children's chronic food refusal. Behavior Therapy, 29, 87-105. 
Table 1

Formulas for Calculation of Treatment Integrity in Preference Assessment

Proportion of correctly labeled foods

following the start of a trial

Proportion of spoon prompts (distance

from mouth) of foods following child's

indication of preference $\underline{\text { Intervals scored with correct instances of labeling foods }}$

Number of presentations of foods

Intervals scored with spoon prompts

Intervals scored with spoon prompts following child's indication of preference

Intervals scored with indications of preference for each food item

Proportion of removal of foods contingent upon non-acceptance within $5 \mathrm{~s}$
Intervals scored with spoon removals when the child does not accept within $5 \mathrm{~s}$

Intervals scored with removals of foods presented

Intervals scored with spoon removals when the child does not accept within $5 \mathrm{~s}$

Intervals scored with non-acceptance within $5 \mathrm{~s}$ 
Table 2

Proportions of Treatment Integrity in Preference Assessment per Child

\begin{tabular}{lcccc}
\hline & Greg & Maggie & Sally & \\
\hline Proportion of correctly labeled & & & & \\
foods following the start of a & 1.0 & 1.0 & 1.0 & 1.0 \\
trial & & &
\end{tabular}

Proportion of spoon prompts

(distance from mouth) of

foods following child's

indication of preference

Proportion of removal of

foods contingent upon non- 
Table 3

Formulas for Calculation of Treatment Integrity in Contextual Variable Evaluation

Proportion of specific prompts delivered

after start of trial
Intervals scored with specific prompts delivered

Number of trials

Proportion of initial spoon prompts

delivered after start of trial

Intervals scored with initial spoon prompts delivered

Number of trials

Proportion of attention deliveries following

Intervals scored with attention deliveries following interruptions or expulsions

interrupted, expelled bites of food

Intervals scored with attention deliveries

Intervals scored with attention deliveries following interruptions or expulsions

Intervals scored with interruptions or expulsions 
Table 4

Proportions of Treatment Integrity in Contextual Variable Evaluation per Child

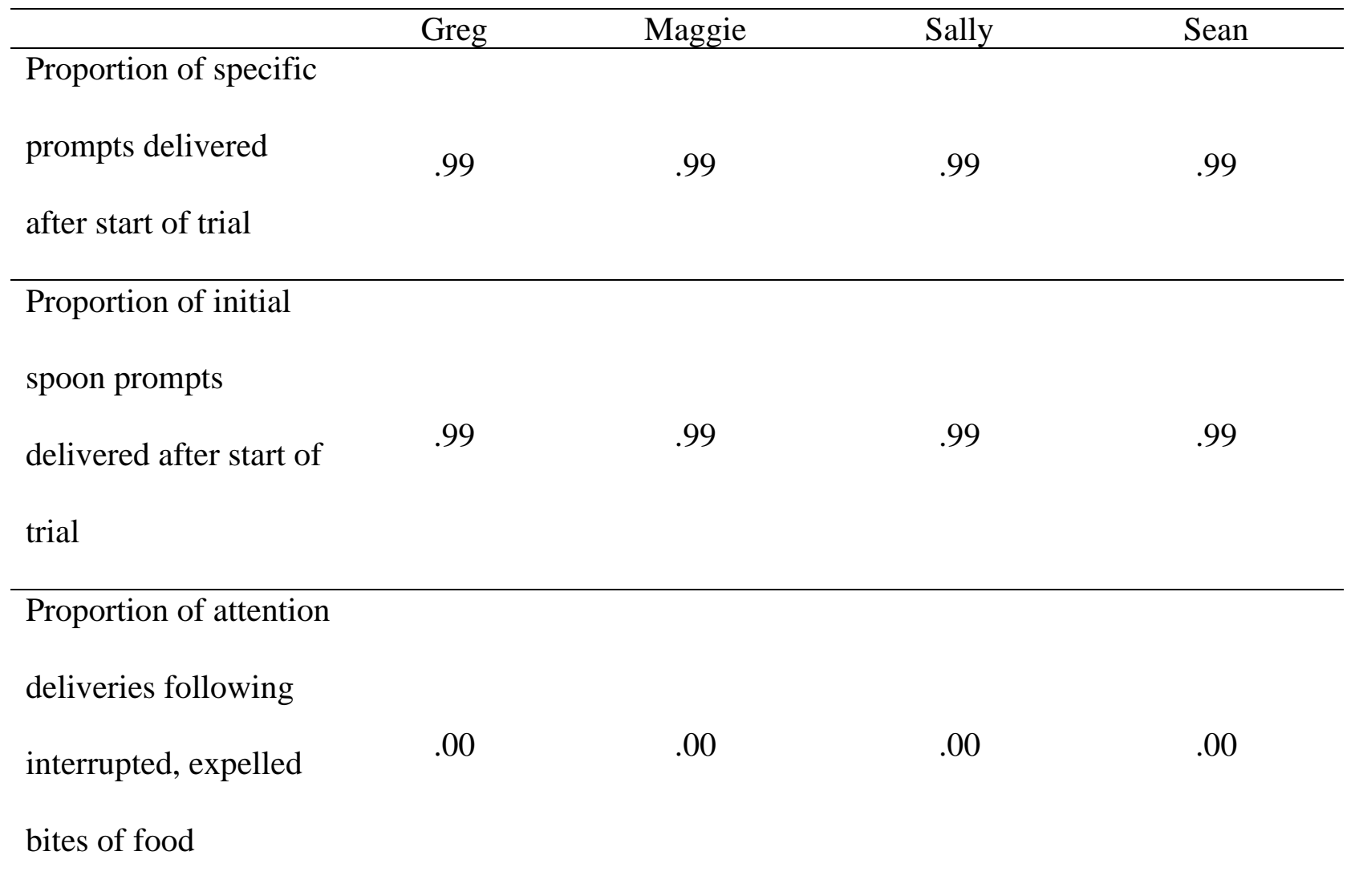




\section{Figure Captions}

Figure 1. Proportion of accepted bites not expelled per session during the preference assessment. Figure 2. Upper panel—Mean accepted bites not expelled per minute for Greg. Second panel— Mean proportion of intervals scored with interruptions and other behavior for Greg. Third panel-Mean proportion of intervals scored with crying for Greg. Bottom panel-Mean responses per minute of negative vocalizations for Greg.

Figure 3. Upper left panel-Accepted bites not expelled per minute per session for Greg. Upper right panel—Proportion of intervals scored with interruptions and other behavior per session for Greg. Bottom left panel— Proportion of intervals scored with crying per session for Greg. Bottom right panel—Negative vocalizations per minute per session for Greg.

Figure 4. Upper panel—Mean accepted bites not expelled per minute for Maggie. Middle panel—Mean proportion of intervals scored with interruptions and other behavior for Maggie. Bottom panel-Mean proportion of intervals scored with crying for Maggie.

Figure 5. Upper left panel-Accepted bites not expelled per minute per each session for Maggie. Upper right panel—Proportion of intervals scored with interruptions and other behavior per session for Maggie. Bottom panel— Proportion of intervals scored with crying per session for Maggie.

Figure 6. Upper panel—Mean tasted of bites not expelled per minute for Sally. Second panel— Mean proportion of intervals scored with interruptions and other behavior for Sally. Third panel—Mean proportion of intervals scored with crying for Sally. Bottom panel—Mean disrobings per minute.

Figure 7. Upper left panel—Bites tasted per minute per session for Sally. Upper right panel— Proportion of intervals scored with interruptions and other behavior per session for Sally. Bottom 
left panel— Proportion of intervals scored with crying per session for Sally. Bottom right panel—Disrobings per minute per session for Sally.

Figure 8. Top panel—Mean accepted bites not expelled per minute for Sean. Middle panel— Mean proportion of intervals scored with interruptions and other behavior for Sean. Lower panel-Mean proportion of intervals scored with crying for Sean.

Figure 9. Upper left panel—Accepted bites not expelled per minute per each session for Sean. Upper right panel—Proportion of intervals scored with interruptions and other behavior per session for Sean. Bottom panel— Proportion of intervals scored with crying per session for Sean. 

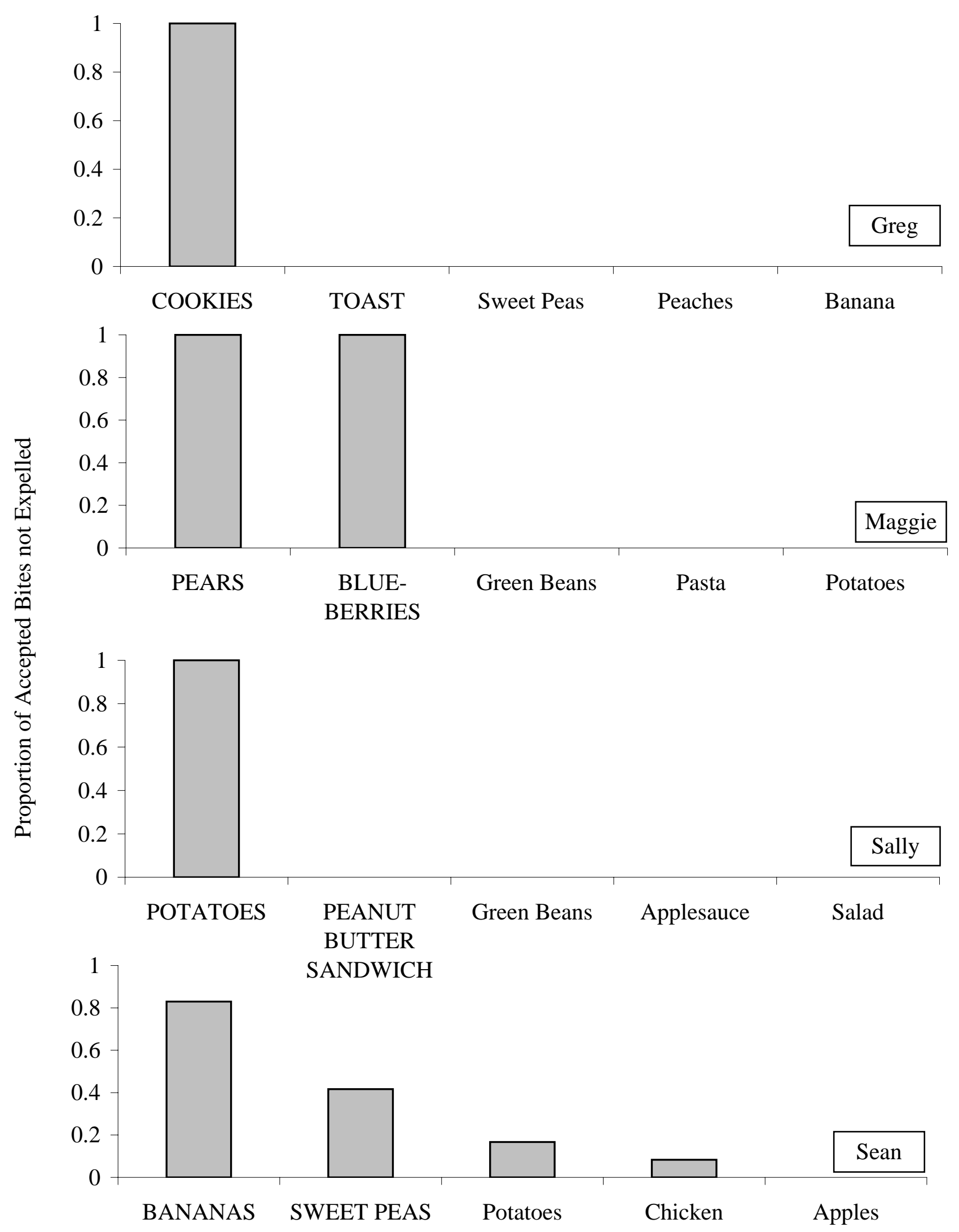

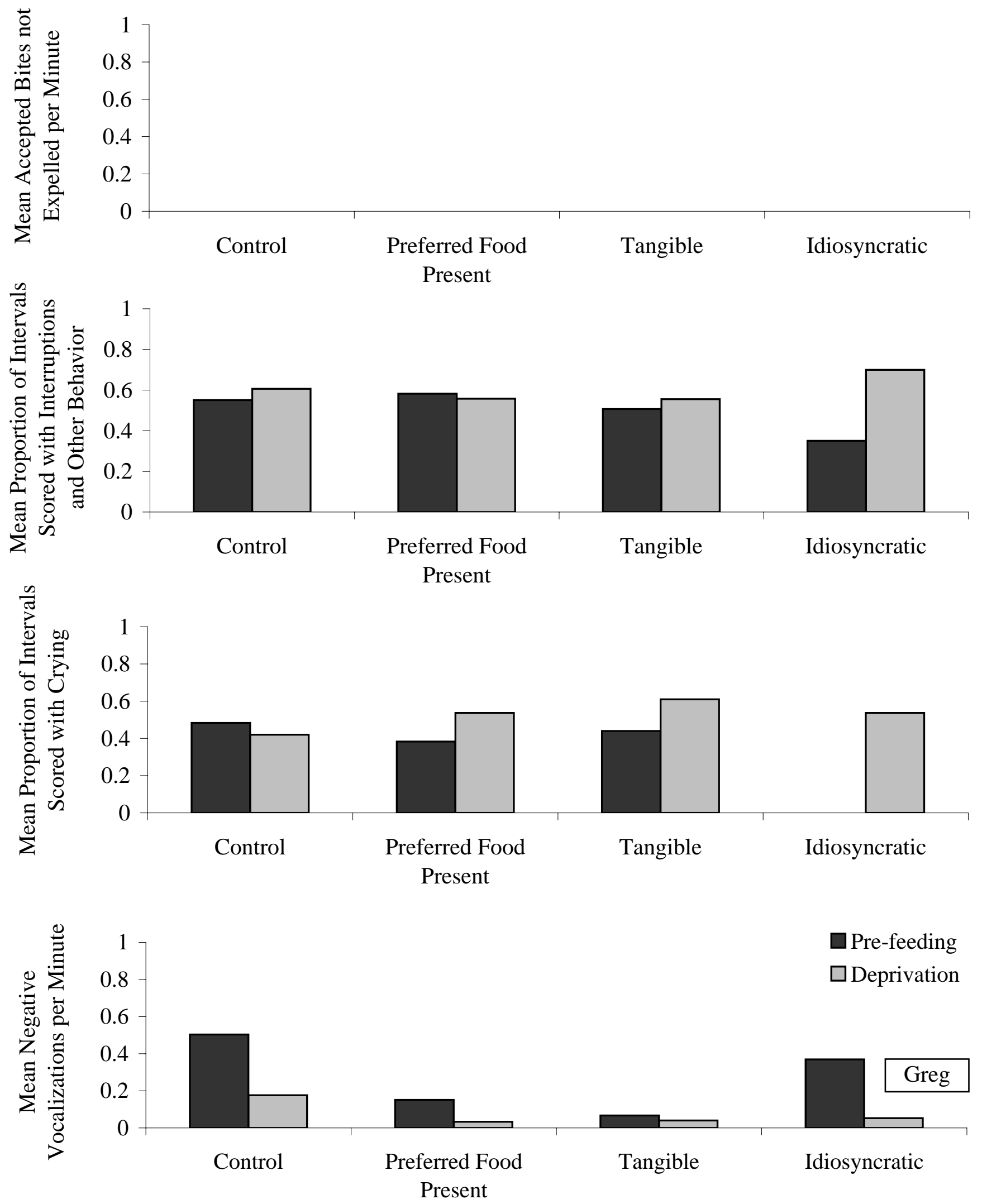

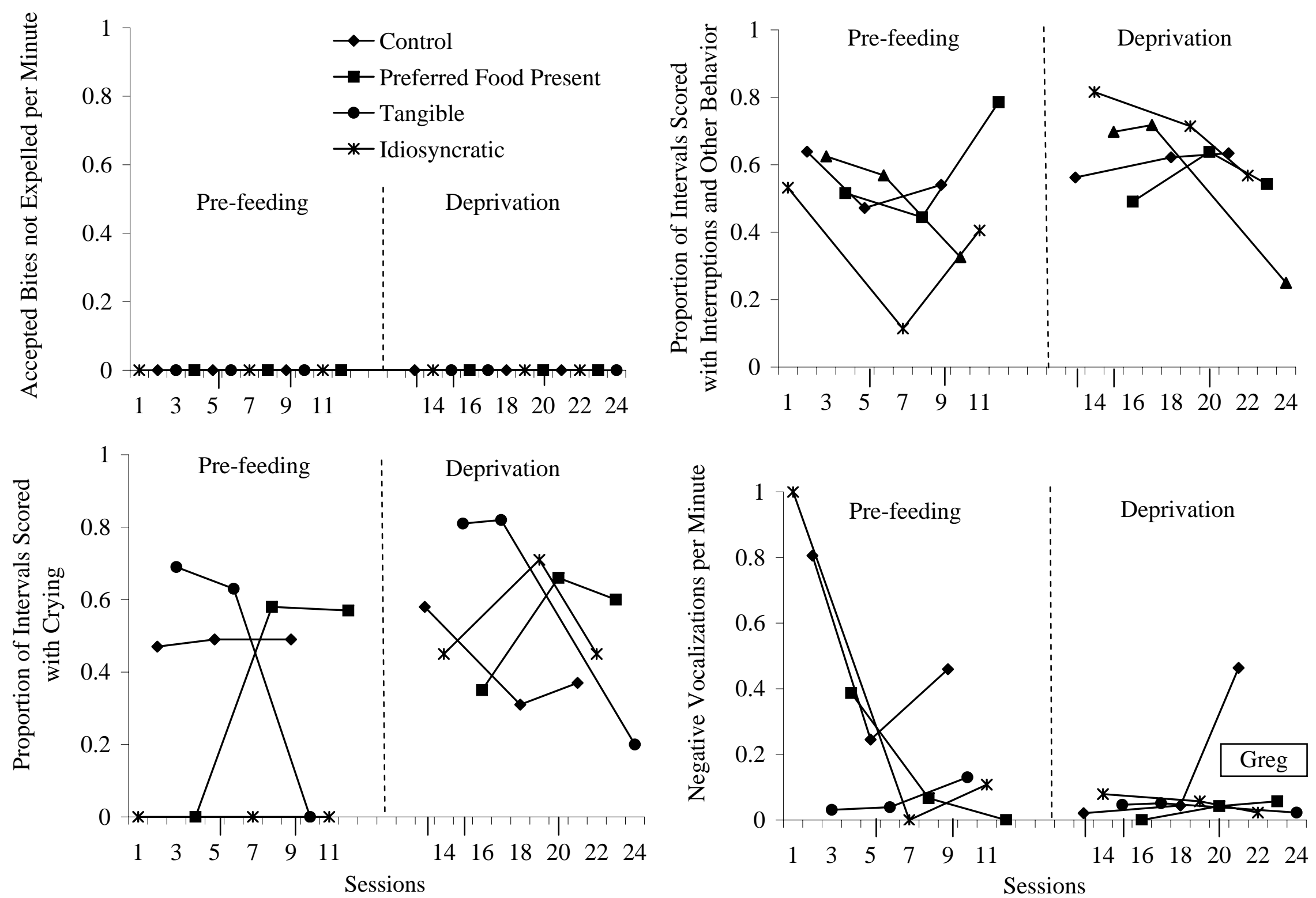

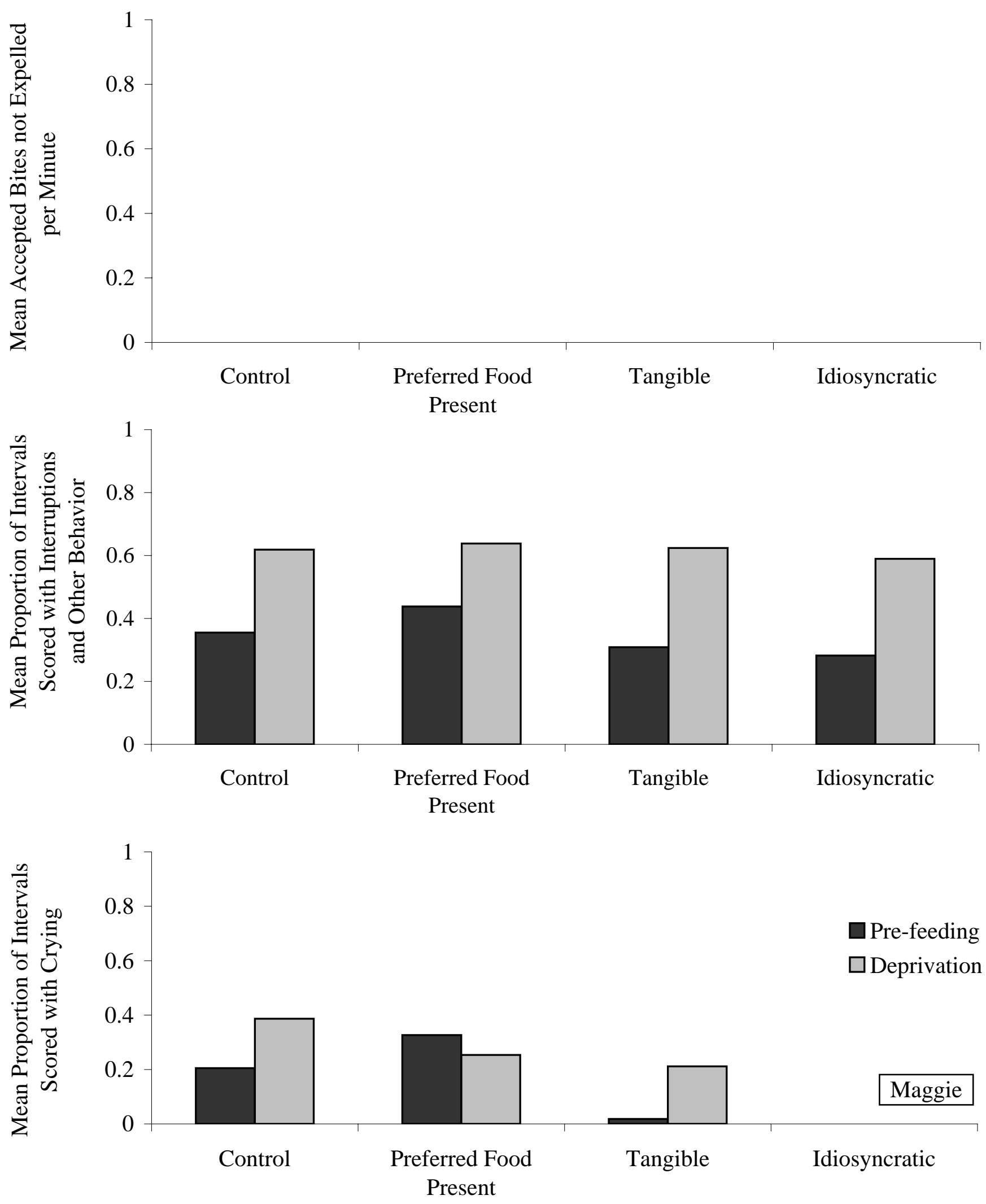

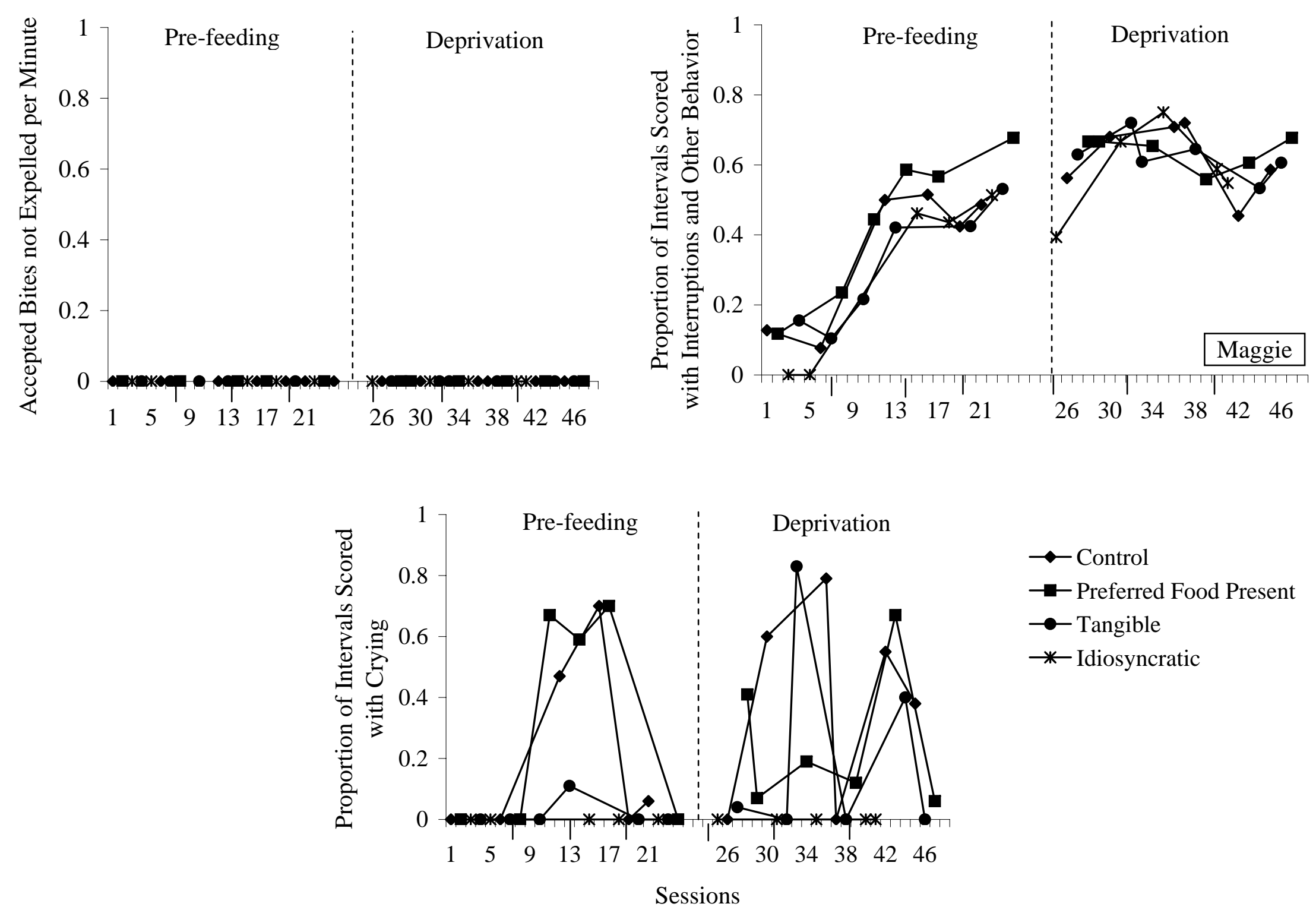

$\neg$ Control

$\rightarrow-$ Preferred Food Present

$\rightarrow$ Tangible

* Idiosyncratic

Sessions 

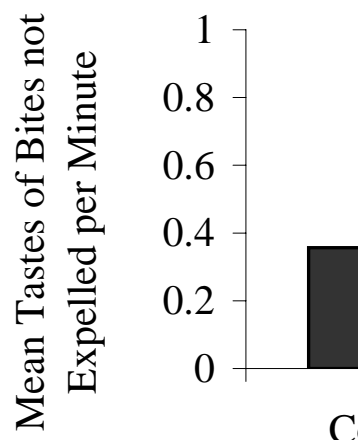

Contro
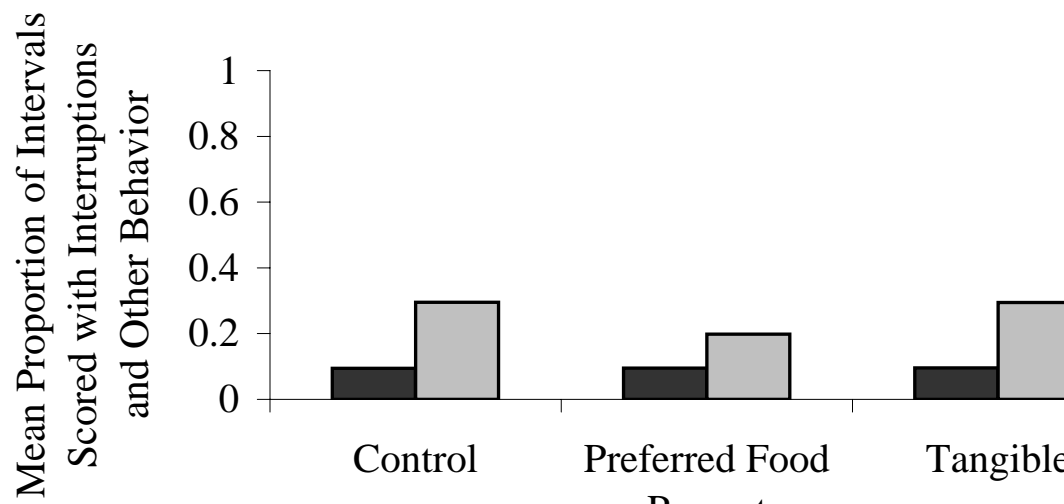

Control

Preferred Food

Present

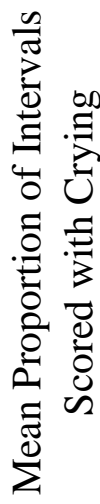

$$
\left.\begin{array}{r}
1 \\
0.8 \\
0.6 \\
0.4 \\
0.2 \\
0
\end{array}\right]
$$
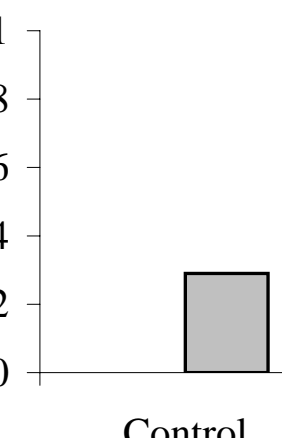

Control

Preferred Food

Present

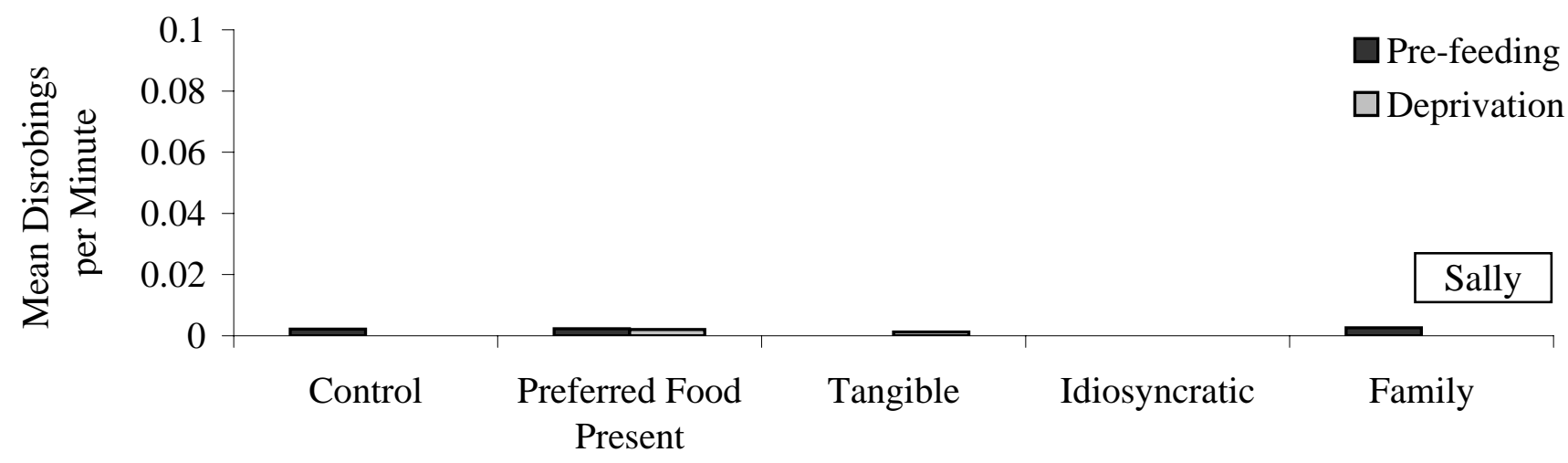



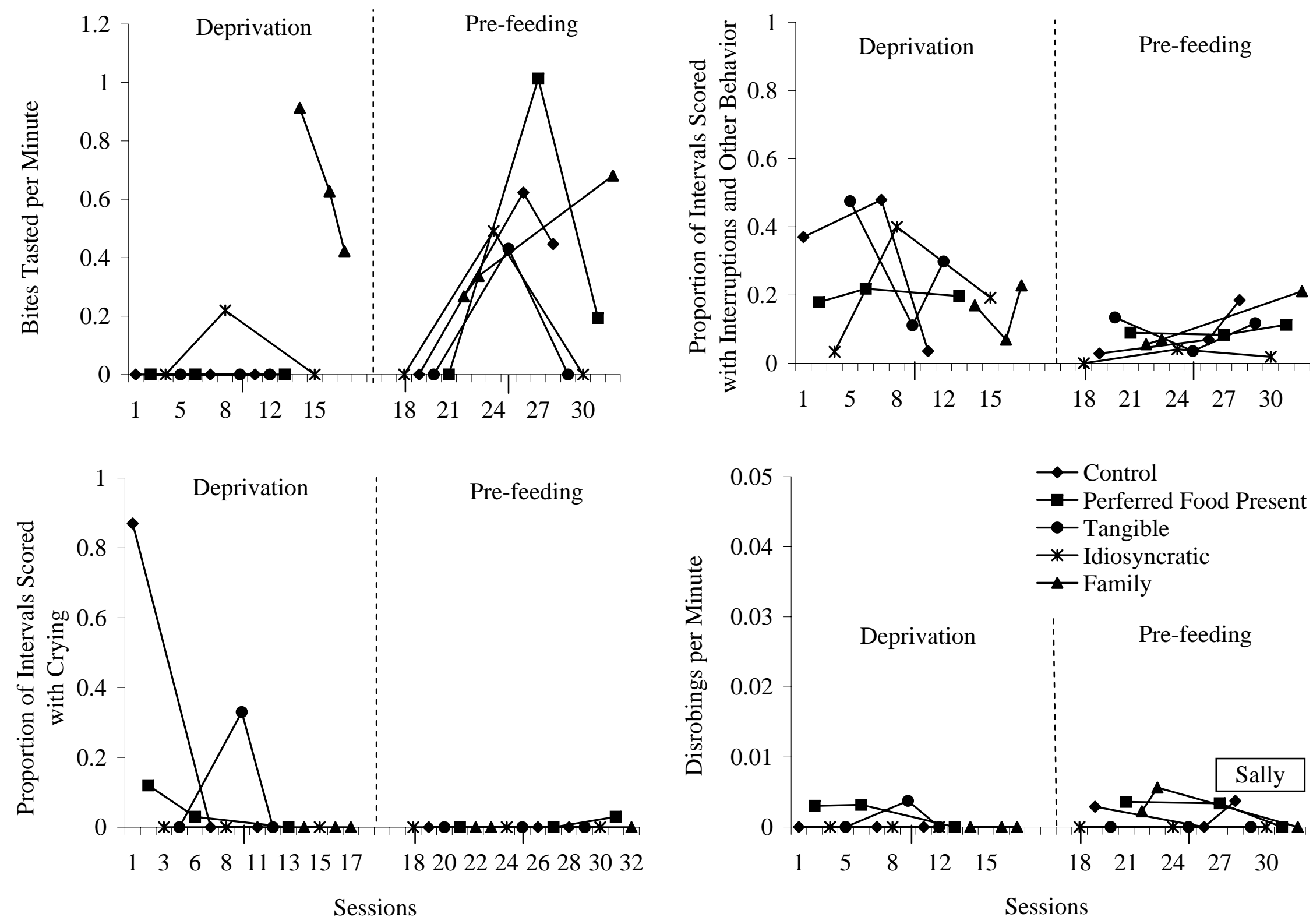

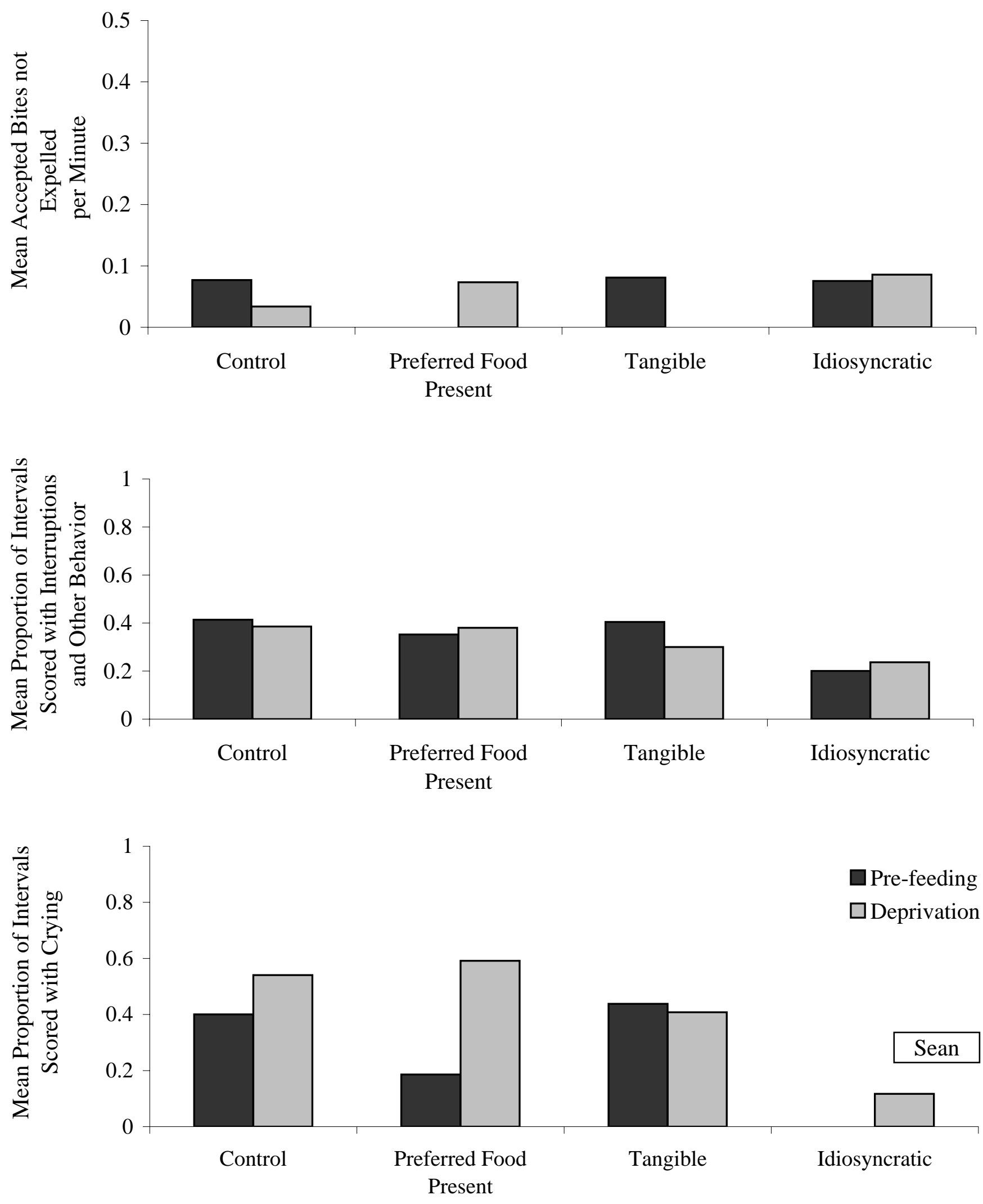

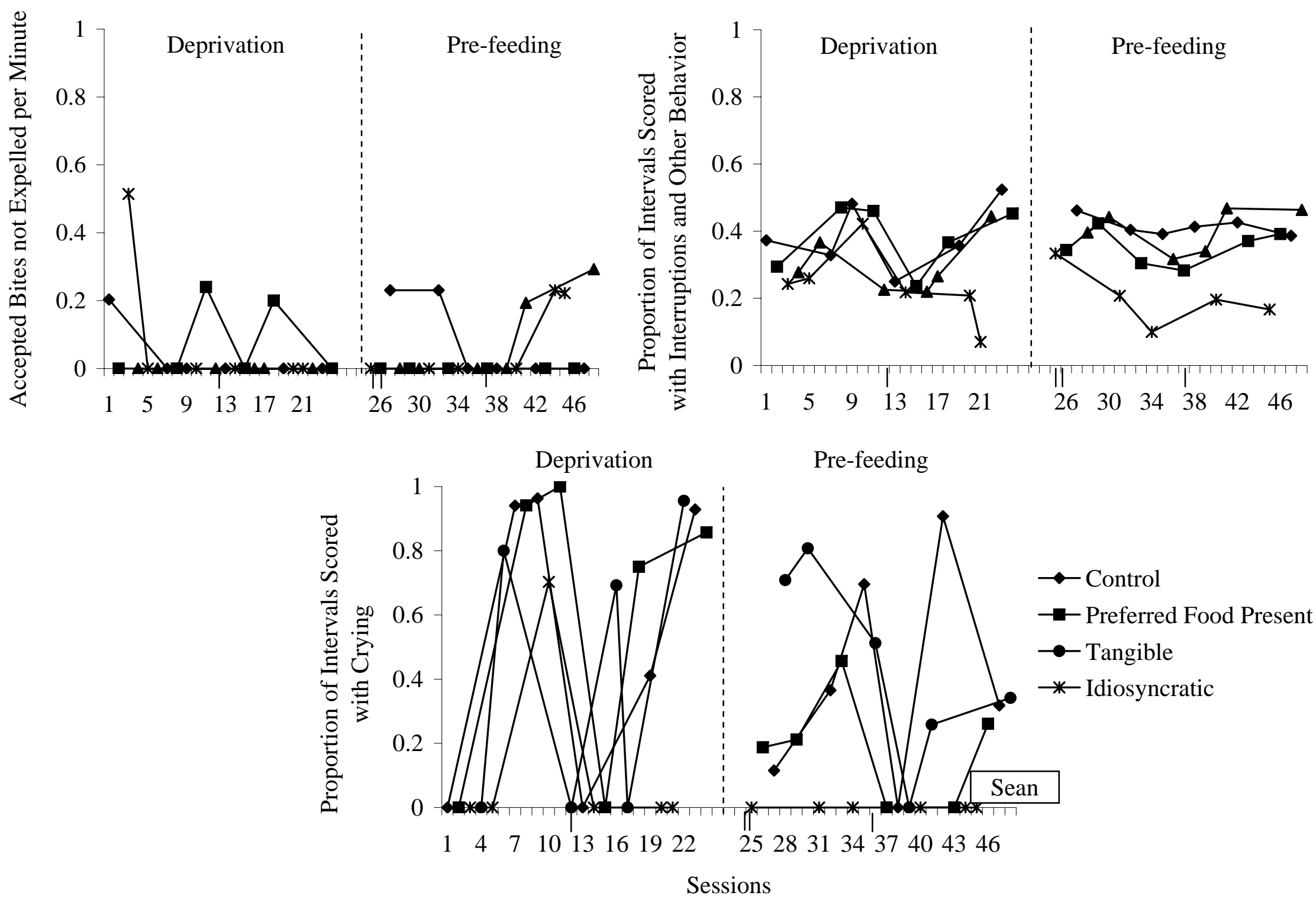